\title{
Presentación
}

\section{¿Mujeres al margen? Estudios empíricos en trabajo y derecho}

Este proyecto surgió a partir de la reunión de tres profesoras discutiendo sobre sus tesis doctorales. Las tres estábamos interesadas en adelantar investigaciones multidisciplinares que, por medio de la investigación empírica, dieran cuenta de las condiciones de trabajo de mujeres en sectores populares en Colombia. Natalia estaba investigando el trabajo formal e informal en confección; Laura se ocupaba de evaluar las condiciones de trabajo de quienes se la rebuscan en la calle y Lina estaba realizando una investigación sobre mujeres mototaxistas en Barranquilla. Este encuentro tuvo mucho de fortuito. Aunque las tres estudiamos derecho en la misma universidad, nuestros programas doctorales nos llevaron por distintos caminos y enfoques temáticos, para luego reencontrarnos política, metodológica y teóricamente en una empresa poco común en el mundo de la academia jurídica: estudios empíricos en derecho, mujeres y trabajo.

En algunas de nuestras conversaciones posteriores nos preguntamos por otros investigadores que estuvieran trabajando en proyectos con enfoques similares a los nuestros. Nuestro reto sería persuadirlos para pensar en una publicación conjunta que pusiera en diálogo esfuerzos que, de lo contrario, resultarían dispersos. No fue fácil porque estábamos buscando personas con formación jurídica que hubieran asumido el reto de adelantar investigación empírica, tan difícil, dispendiosa y escasamente financiada, y que además tuvieran resultados nuevos que quisieran publicar. Para nuestra fortuna, nos encontramos con un grupo de autores que seguro no serán los únicos, pero que dadas las limitaciones de tiempo y recursos que enfrentaba nuestro proyecto, consideramos suficientemente representativos. Sus trabajos daban cuenta de mujeres de distintos niveles socioeconómicos, en distintas zonas del país, que desempeñaban distintas ocupaciones para ganarse la vida, tanto en sectores rurales como urbanos y en trabajos formales e informales.

Los textos que presentamos en este número especial explotan excepcionalmente esa diversidad. María Victoria Castro y Lina Buchely analizan el caso de las muje- 
res mototaxistas que trabajan informalmente en Barranquilla a través del lente de sus experiencias subjetivas. Juan M. Amaya-Castro y Daniela Palacio-Rodríguez exploran la economía del tiempo de trabajadoras que ocupan cargos administrativos en el noroccidente de Bogotá para entender sus dinámicas y estrategias espacio-temporales. Valentina Montoya estudia el caso de las trabajadoras domésticas sindicalizadas y no sindicalizadas en Medellín para describir sus logros y retos en términos de su interacción con el gobierno nacional y local en materia de derechos laborales. Javier A. Pineda D. estudia a enfermeras y auxiliares de enfermería en Bogotá que realizan trabajo de cuidado remunerado con personas de la tercera edad para describir sus condiciones de trabajo y plantear el reto de la desvalorización del trabajo de cuidado institucional.

Por su parte, Yenny Carolina Ramírez-Suárez y Fabián Esteban Pinzón-Díaz estudian a las mujeres de la comuna 13 de Medellín, quienes desde la cultura hip-hop desarrollan prácticas que, aunque inscritas en condiciones de precariedad, resignifican la vida en términos políticos, sociales y culturales. María Carolina Olarte-Olarte y Guisella Lara-Veloza estudian el caso de un grupo de mujeres que hicieron la apuesta de convertirse en trabajadoras cooperativas de una huerta en Madrid, Cundinamarca, como fuente de sustento alimentario y también como proyecto de educación popular para el trabajo digno, la apropiación del territorio y el cuidado de sus cuerpos afectados por la floricultura. Astrid Sánchez-Mejía y Juliana Morad-Acero hacen la única contribución cuantitativa en este número especial. En su investigación, las autoras estudian la historia laboral de mujeres reclusas antes de ser detenidas, así como sus expectativas para regresar al mercado laboral después de su paso por la cárcel.

Finalmente, Natalia Ramírez-Bustamante describe el paso del trabajo formal al trabajo informal en confección como consecuencia de dos tipos de tensiones. Por una parte, como el efecto de distintos mecanismos discriminatorios en las empresas formales y, por otra, como el resultado de la inflexibilidad de estas, lo que les impide a las trabajadoras la articulación entre el trabajo de cuidado y el trabajo económicamente productivo. Laura Porras-Santanilla y Andrés Rodríguez-Morales estudian las formas de conciliar las tareas productivas y reproductivas de vendedoras ambulantes que tienen hijos entre cero y cinco años en Bogotá, para argumentar que la oferta institucional pública de cuidado de la ciudad no tiene en cuenta sus necesidades al momento de crear mecanismos para conciliar la tensión entre familia y trabajo.

Todos los autores presentaron versiones preliminares de estos textos en un taller que se realizó el 15 y 16 de agosto de 2018 en la Universidad del Rosario, gracias al apoyo económico de la Facultad de Jurisprudencia y del CIJUS de la Universidad de los Andes. Participaron como comentaristas invitadas las profesoras Katherine Lippel (Canada Research Chair on Occupational Health and Safety Law) y Friederike 
Fleischer, profesora asociada del Departamento de Antropología de la Universidad de los Andes, quienes facilitaron la identificación de temas transversales e hilos comunicantes, y mejoraron en general la calidad de todos los textos. Por ello les estamos infinitamente agradecidas. Las discusiones durante los dos días del taller fueron tan variadas y fructíferas que los participantes se comprometieron a escribir versiones que incorporaran la retroalimentación de las comentaristas y los demás coautores. Finalmente, queremos agradecer a la Escuela Nacional Sindical que organiza anualmente el Concurso Latinoamericano de Fotografía Documental "Los trabajos y los días". Gracias a la generosidad de la Escuela, este número especial cuenta con cinco fotografías seleccionadas del concurso en sus versiones 2016 y 2018, que enuncian parte de la poética y diversidad de roles que enfrentan las trabajadoras colombianas.

Ahora bien, hay tres temas transversales que entretejen el trabajo de todas las contribuciones a este número especial. El primero es la pregunta por el trabajo: qué es y qué no es trabajo, y qué ganamos o perdemos al usar esa etiqueta. Por ejemplo, existen formas de trabajo socialmente invisibles, es decir, que implican una dedicación de tiempo y esfuerzo organizativo y comunitario de quienes las desarrollan y consideran que sus actividades constituyen trabajo, a pesar de que no necesariamente son socialmente reconocidas ni visibles para el derecho. Esta clase de trabajos, dentro de los lugares sociales que analizan los artículos que reunimos en este número, están altamente feminizados. Es decir, son las mujeres las que se dedican a esos "trabajos invisibles", ilegibles para las formas jurídicas, pero transformadores para el cotidiano social. Nos referimos por ejemplo al tipo de actividades que describen los trabajos de María Carolina Olarte-Olarte y Guisella Lara-Veloza (trabajo político) y Yenny Carolina Ramírez-Suárez y Fabián Esteban Pinzón-Díaz (trabajo organizativo y artístico para comunidades vulnerables). Otros ejemplos de relativa invisibilización en el derecho son el trabajo reproductivo y el trabajo informal, que, así como los anteriores, son muy demandantes en términos de tiempo y escasamente o no remunerados.

El segundo tema transversal es la escasez o pobreza de tiempo al que se enfrentan los distintos grupos de mujeres estudiados en los artículos que componen este número especial, así como las tácticas que ellas despliegan para subvertir esas dificultades: atajos, estrategias de movilidad múltiples y epistemologías logísticas complejas que las llevan a simplificar el tiempo que invierten en "llegar" al trabajo. Se concentran en este tema, que suele recibir poca atención pese a su importancia, los artículos de Juan M. Amaya-Castro y Daniela Palacio-Rodríguez por un lado, y Valentina Montoya, Lina Buchely y María Victoria Castro por otro. Sus hallazgos indican que, en general, los trabajadores invierten un número muy importante de horas diarias en el transporte público en los trayectos hogar/trabajo/hogar, tiempo que no es re- 
munerado y que es comparativamente mayor en el caso de las mujeres trabajadoras que en el de los hombres, dado que sus trayectos y frecuencias no coinciden con las ciudades tipo que imaginaron los urbanistas. Estos hallazgos también nos dejan ver cómo las mujeres han enfrentado, de formas más o menos exitosas, esa situación.

Esta dimensión pone sobre la mesa el tema de los costos indirectos del trabajo y cómo se distribuyen socialmente sus cargas. El diseño urbano de las rutas de transporte, los tipos de trabajo y mecanismos de movilidad disponibles (junto con sus rutas y horarios) y los accesorios necesarios para el ejercicio del empleo son elementos marginales en las preguntas tradicionales sobre el derecho laboral, pero centrales en análisis empíricos que muestran su rol crítico en las experiencias laborales de las mujeres. Desde otra perspectiva, pero también con respecto a la pobreza de tiempo, el trabajo de Natalia Ramírez-Bustamante subraya los efectos de los largos horarios y la inflexibilidad de las jornadas laborales en el trabajo formal en confección, y cómo estos operan como detonantes para que algunas mujeres migren del trabajo formal al informal. De aquí, entonces, que esta apuesta subraye el carácter contextualizado (conectado con espacios, tiempos y subjetividades) del trabajo como una de sus conclusiones principales.

Finalmente, el tercer tema transversal en este número es el trabajo informal, que, sin proponérnoslo, aparece en la mayoría de contribuciones. Pero, en realidad, no se trata de una simple coincidencia. Según las cifras del DANE, para el trimestre móvil de febrero a abril de 2019, en las 23 ciudades y áreas metropolitanas el mercado laboral colombiano está fraccionado entre un $47,7 \%$ de trabajo informal versus un $52,3 \%$ de trabajo formal y, adicionalmente, la participación de las mujeres en la informalidad es más alta que la de los hombres, pese a que las mujeres, en promedio, acumulan un mayor número de años de educación que ellos. Los hallazgos de Ramírez-Bustamante y de Laura Porras-Santanilla y Andrés Rodríguez-Morales muestran, por ejemplo, que el trabajo formal es poco flexible y dificulta la articulación entre el trabajo de cuidado y el económicamente productivo, lo que obliga a muchas mujeres a "optar" por la informalidad en búsqueda de poder cumplir con funciones de cuidado que les siguen siendo atribuidas de manera desigual. Estas "decisiones" traen consigo un costo, pues la posibilidad de acceder a prestaciones económicas ocasionadas por accidentes laborales, invalidez, vejez y muerte son mínimas, cuando no nulas. Esta situación pone de presente una vez más lo inconveniente que resulta atar las prestaciones de seguridad social únicamente al vínculo laboral formal, pero también ilumina agencias de las mujeres que gestionan sus realidades de trabajo en escenarios densos, cuyas cargas de trabajo destruyen la dicotomía productivo/ reproductivo y hablan de dinámicas de acción y trabajo más complejas de lo que el derecho laboral imagina. 
La idea de trabajo digno no es una receta que funcione igual para todo el mundo. A nuestro juicio, se trata de un concepto contingente con variables diversas y difíciles de discutir que aún no están resueltas. No tenemos claro qué es trabajo, tampoco qué es trabajo digno, ni qué ganamos y qué perdemos con llamar algo trabajo. Tampoco tenemos claro el alcance y los límites del derecho laboral, ni sabemos si esperar más de él y de qué formas. ¿Las condiciones materiales de vida de las mujeres protagonistas de estos textos mejorarían con reformas jurídicas que ampliaran el espectro del derecho laboral para incluirlas? Esperamos que las investigaciones que se desarrollan en los artículos que componen esta revista estén en el camino de resolver esta y otras preguntas.

\section{Natalia Ramírez-Bustamante}

(Universidad de los Andes)

\section{Laura Porras-Santanilla}

(Universidad de los Andes)

\section{Lina Buchely}

(Universidad Icesi)

Editoras invitadas

Bogotá y Cali, agosto de 2019 



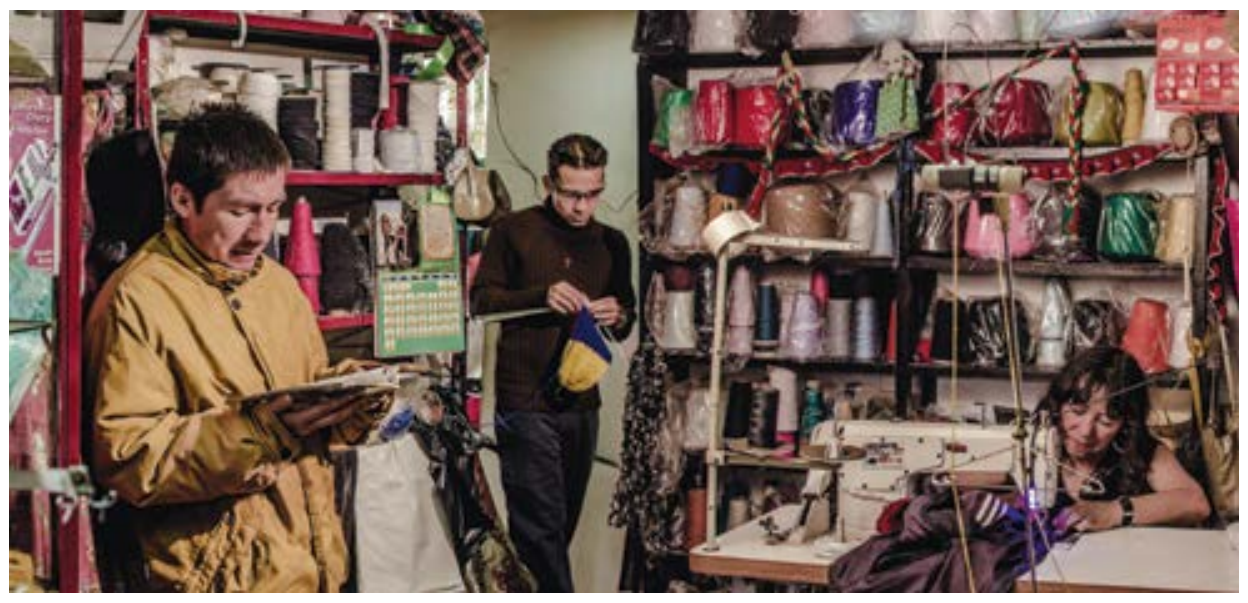

Invisibles 4

Andrés Millán 


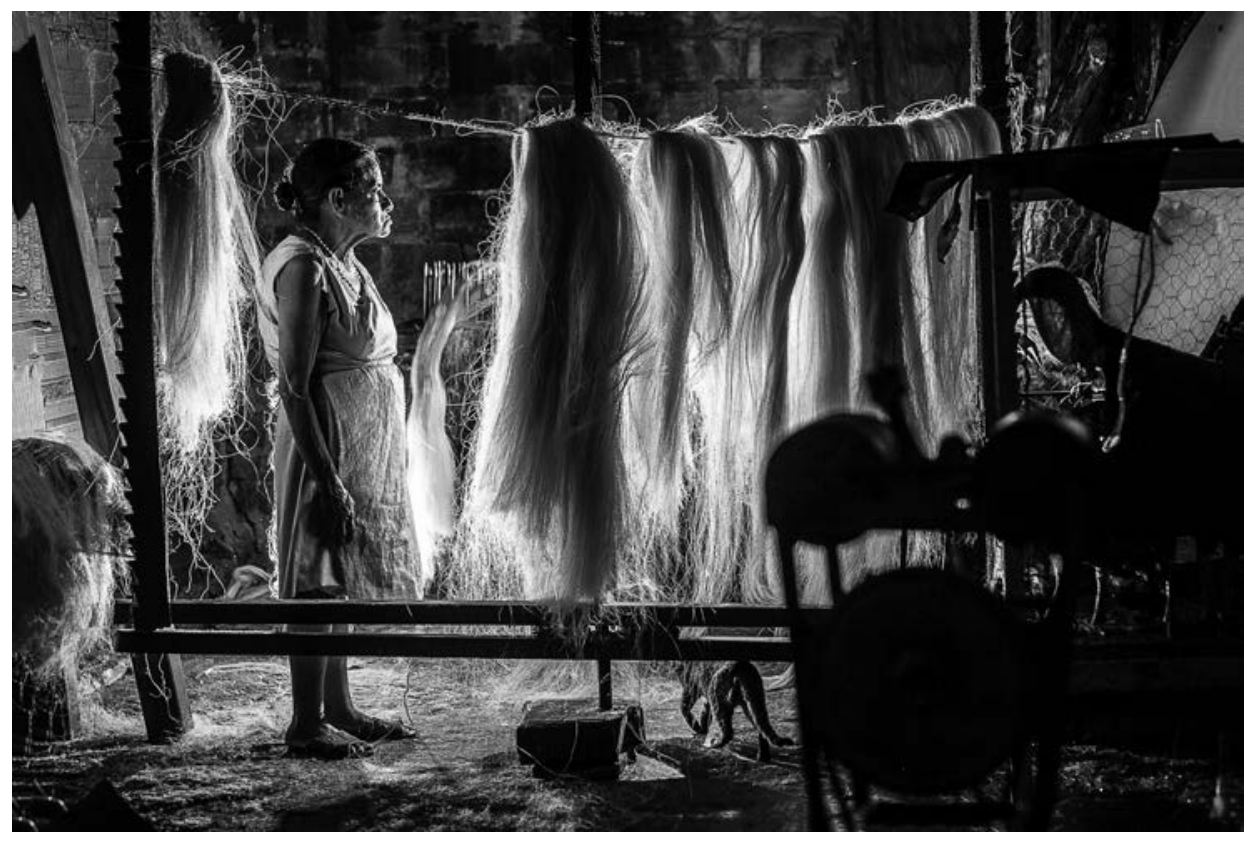

Fique 1

Daniel Ricardo López Gútierrez 


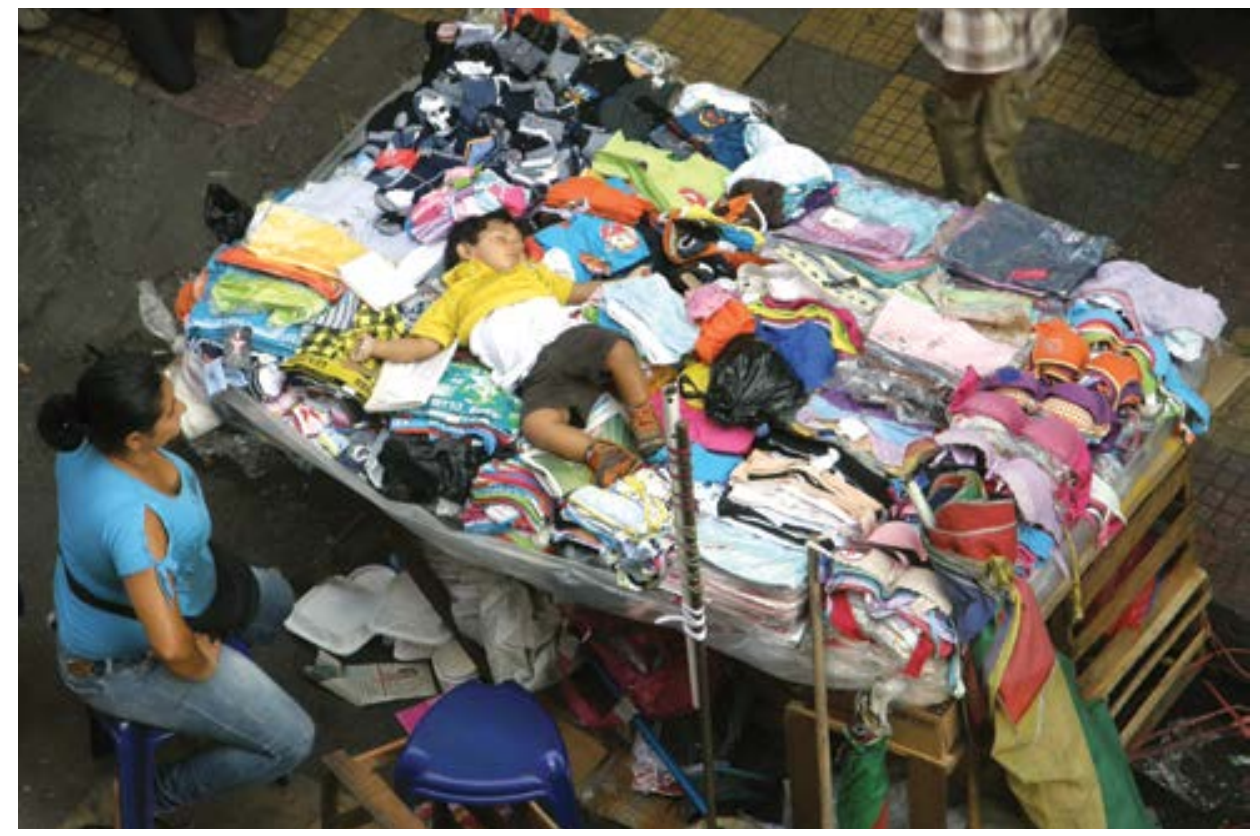

La oficina

Javier Gutiérrez 


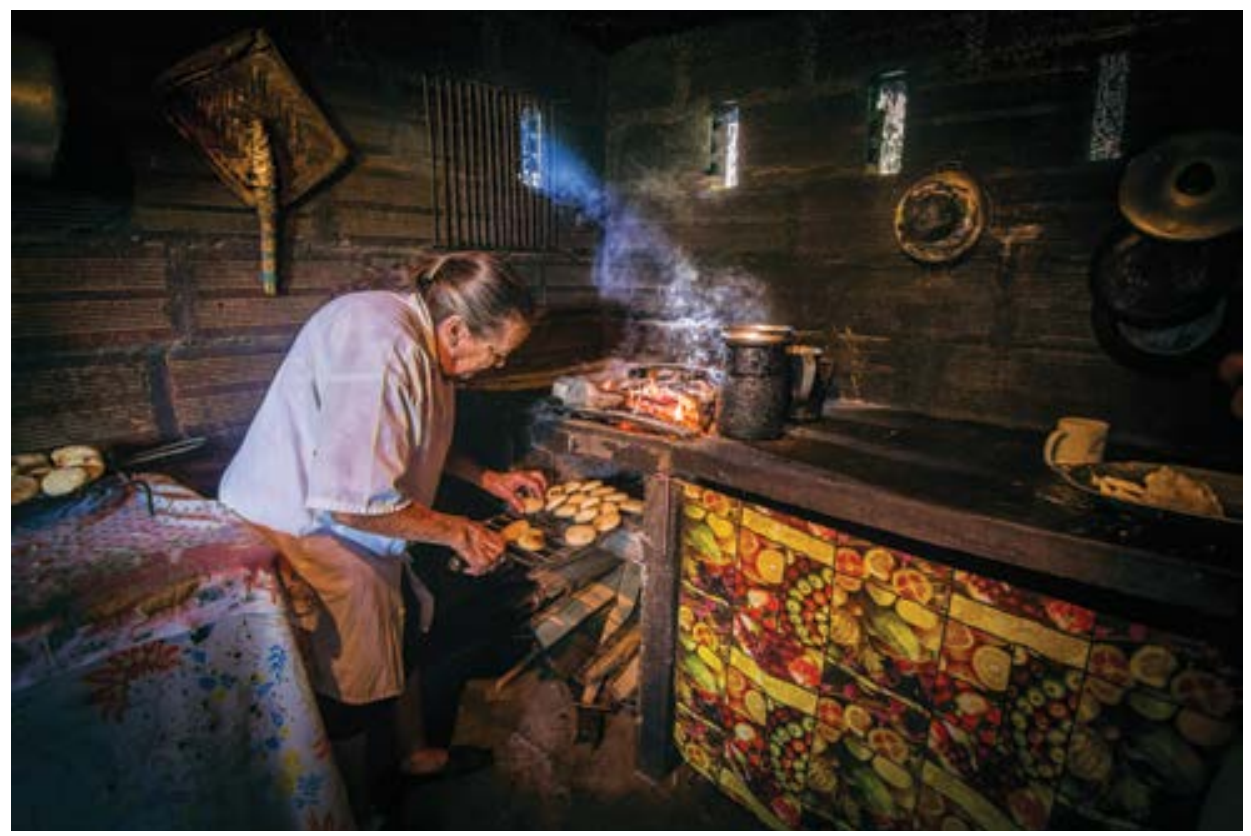

Tradición de las arepas paisas

Fabián Rendón Morales 


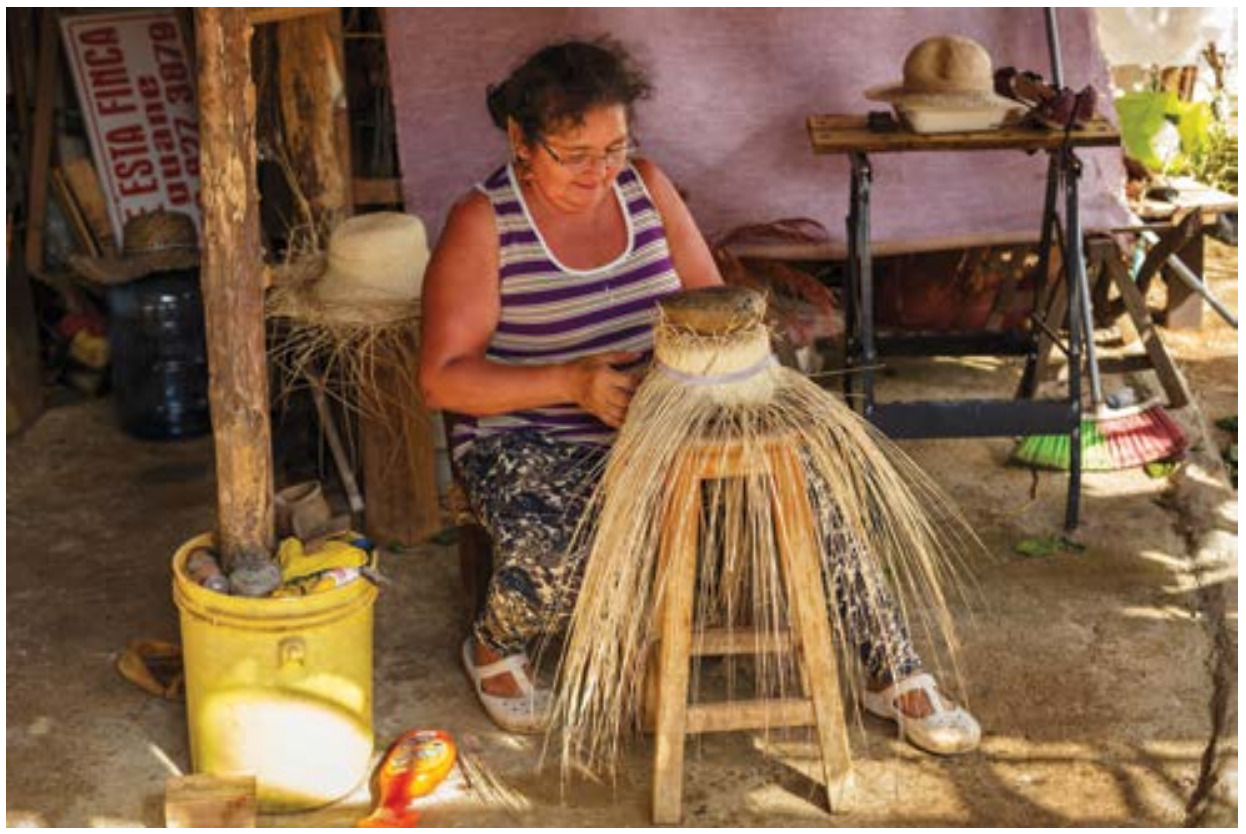

Saber de iraca 1

Javier Jiménez Corzo 
Fotografías cortesía de:

Concurso Latinoamericano de Fotografía Documental "Los trabajos y los días"

Escuela Nacional Sindical 